\title{
False Alarm Detection in Wireless Body Sensor Network using Adaptive and Intelligent Approach
}

\author{
Binoy Barman Shubha \\ Department of Information and Communication Technology \\ Mawlana Bhashani Science and Technology University
}

\author{
Sajjad Waheed \\ Department of Information and Communication Technology \\ Mawlana Bhashani Science and Technology University
}

\author{
Kazi Sadlil Rhythom \\ Department of Information and Communication Technology \\ Mawlana Bhashani Science and Technology University
}

\begin{abstract}
False Alarm is one kind of faulty measuring process which can create unusual intervention over healthcare personnel. In our paper, we proposed an Adapting and Intelligent approach to help detecting false alarm in WBSN (Wireless Body Sensor Network). The practical implementation of our work is able to explore the challenges over our false alarm detection architecture. Our system is able to detect the false alarm thats occurred in the patient monitoring sensor data transmission and also have capability to minimize this faulty measure. Moreover, our approach over false alarm detection afforded by WBSN can improve the primitive patient monitoring system as well as the quality of wireless based healthcare service. We proposed two approaches for detection false alarm in the wireless body sensor network. One is MD (Mahalanobis Distance) and another is Dynamic Threshold Calculation method.
\end{abstract}

\section{Keywords}

False Alarm, WBSN (Wireless Body Sensor Network), MD, Dynamic Threshold, Truetime.

\section{INTRODUCTION}

Wireless Sensor Network (WSN) includes a number of battery powered sensor nodes, endowed with physical sensing capabilities, limited processing and memory, and short range radio communication. This sensor networks are used in numerous application domain. The sensing nodes have a sensing device that acquires data from the physical environment. It also has a processing subsystem for local data processing and storage and a wireless communication module. This is one kind of distributed wireless sensor networks not only energy but also processing constraints. In todays advanced world most of the low power hardware architecture and also most communication protocols are expressed the use of WSNs in various high data intensive applications. In wireless sensing and networking technologies, these types of progression for variant applications are obviously the key enablers for the effective integration of physical as well as cyber worlds. As an application, we can use wireless sensor network in the medical domain, which can develop the arrangements [6] as well as the management of healthcare services. The resource constrained devices are small enough as a wireless medical sensor, its also capable of collecting variant physiological parameters, like; Heart Rate (HR), Pulse, Oxygen Saturation (SpO2), Respiration and Blood Pressure (BP). These types of sensed data can provide a number of information which are valuable for doctors, nurse and also for particular care givers. These help them to determine the overall medical condition of the subject. Now-a-days for a long period of time, hospital monitoring is very costly. So there has a stable option, which is to keep the non-emergency subjects in the patents home and make a process to continue monitoring using variant medical sensor [24]. There are a number of medical sensors, like; MICAz [1], TelosB [5], Shimmer [10], and IRIS [36]. These are more flexible in some special terms of mobility as well as for movement [7]. To ensure the accuracy and reliability of the monitoring data its important to raise an alarm in case of emergency, though for caregiver it is may not possible to present all the time.

In the application of Wireless Sensor Network (WSN), Body Sensor Network (BSN) [31] uses a large number of body sensors for measuring the medical condition of a patient. Due to sensor fault, the collected sensor data may be inaccurate. There are also other factors which can create data inaccuracy such as sensor displacement, transmission interference, malicious data injection and so on. Due to transmission error it is also causing unreliability of sensor data. All of which may generate a large number of false alarms in healthcare application. Those false alarms make a lot of confusion and decrease the efficiency of an overall healthcare process. False alarms have a great negative impact over the health care system. The perfect detection of data inaccuracies at the sensor node is very much important because the collection of faulty resulted data may inject the system as well as can comprise the system, as a result generate many false alarms.

The amount of accumulated data grows over time in case of continuous monitoring. So a fast processing and an alarm generation system required here. The absence of this type of system, the timely detection of emergency medical conditions may not be possible. So there requires a real-time, fast and reliable system in order to improve the quality of medical care by detecting unreliable faulty sensor data, and then identifying as well as isolating potential false alarm, and finally generating a true alarm. A number of false alarm detection systems have been proposed and applied in date. Most of cases for battery operated wireless sensors the resource requirement is not sufficient. In other cases, the centralized approach [34] is not an energy efficient way of routing in WSN and can deplete the sensors energy very quickly. So here, all data need to be transmitted to the sink for processing. In this paper, a novel and efficient false alarm detection system is introduced by using adaptive and intelligent approach. It is essential to analyzing the anomalies to determine whether the anomalous values are indeed faulty or not. They can represent a true medical condition. The decision of generating true and false alarm is made over this analysis. In this paper a novel false alarm detection architecture is proposed and also explored the challenges associated with its practical implementation.

The organization of the paper is as follows. Section II will introduce with some related works with the approach and also give a clear comparison with them. Section III gives an overview about false alarm detection approaches. Section IV and V will give the solution and approaches for the false alarm problems. Section VI and VII will simulate the solution, when VIII will compare the result of the system. Some future works and results will be discussed in section IX and X. 


\section{RELATED WORK}

A number of researchers propose different kinds of approach in order to detect anomalies in medical data. Various medical approaches for WSNs have been proposed over health monitoring. In order to detect patient inactivity in his home as well as trigger an alarm, especially for an immobile patient over a long time [9] an accelerometer-based method was used. Under remote monitoring there also other applications in order to detect an elderly peoples falls. There are also versatile architectures based on medical sensor networks. These are proposed as well as deployed specially for monitoring patients and also for alarm rising for medical emergencies. As an example, in figure 1, we can see that various monitoring activities over a patient. For HR monitoring MEDiSN [19] and CodeBlue [23],[4] can be used, for ECG, SpO2 and pulse, lifeguard [25] can use, again for physiological (pulse and $\mathrm{SpO} 2$ ) and environmental parameters (temperature and light), respiration, pulse oximeter, and blood pressure, AlarmNet [38] and Medical MoteCare[26] can be used. By observation over medical applications we can see that WSNs are available for use [16].

The quality and reliability of WSNs data collection is very poor because of its limited resources. There is a method called data filtering, which can be used for reducing noise level as well as retaining perfect data. It has the ability to remove outliers which can not only change but also can reduce the shape of variation. This is obviously better than only datasets cleaning.

So to detect unusual deviations a normal data model can build

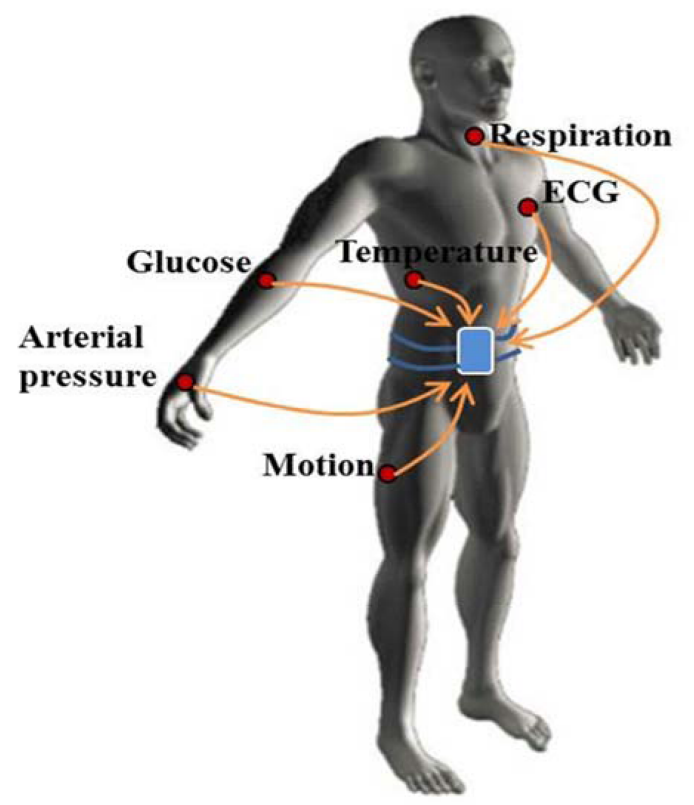

Fig 1. Body Sensor

by using an intrusion detection system based on anomalies. In WSNs versatile approaches has been proposed as well as applied in order to detect abnormal deviations among collected data. There are also approaches for analyzing data accuracy and the ration of false alarm [40], [44]. For classification machining learning algorithm [8] and for clustering data mining [24]1 can apply widely. Like; neural networks, Naive Bayes, decision trees (C4.5) [12], support vector machines (SVMs) [32] [41] [43] [21], self-organizing maps [35], k-means [15], k-nearest neighbor [14], expectation maximization, hierarchical clustering, fuzzy Cmeans, and Gaussian mixture model [37] and so on. In [8] we can see different techniques over classification and clustering is being studied.

In[18], to evaluate the reliability of a WSN over industrial field which belong a large number of sensors logistic regression models including a static threshold was used. But this model is not able to identify the cause of potential loss of reliability. Again on the larger sensor networks a diagnosis method has been proposed, which is based over enhanced C4.5. This is able to answer the overall network accuracy by merging a big spanning tree into local classifiers [12]. Using SUN SPOT a persons physical activity can monitor [42]. A persons physical condition, like; sitting, standing, walking or lying down can be detected by using a Nave Bayes algorithm. But here no type of consideration is included in the corrupted value occurs with the faulty hardware. On the other hand, a system over logistic regression which is based on HR variability can able to make a difference between a persons mental state with stress as well as relaxation states [13].

For data classification, a classifier named SVM is able to gain a high popularity because of its optimum solution and easy numerical comparisons. For anomaly detection in WSNs versatile approaches of SVM has been proposed on [41],[43], [27]. In SVM several nonlinear versions have been investigated to detect a boundary (or hyperplane). In the training phase this boundary can able to encompass the majority over data. After establishing a decision boundary, every new data will be considered as abnormal data on the outside of that boundary.

We know the machine learning algorithm refers a pre-classified training data set. Sometimes this type of data sets may not available in the real world. The meaning of skewed labeled data is there are almost no anomalies in the training data sets. But constructing this type of labeled training set is very expensive as well as very laborious. Researcher tried to resolve this type of problem by using data mining techniques, where abnormal data are grouped in by clustering. In this technique, all of the abnormal data can be distinguished from the normal data. If we compare a normal data clusters size, then we can see that abnormal data are rare here.

In WSNs, to detect outlier a survey of various techniques has been proposed. Like, based on the characteristics of used data set, as there is a comparative guideline of suitable technique [44]. In WSNs, for predicting missing data linear regression was used [39]. So in order to detect anomaly in WSNs various approaches were used, like; linear least squares estimation, auto regressive integrated moving average, hidden Markov model, etc. [33]. Assuming neighbor nodes monitor the same attributes to identify insider malicious sensor, a distance-based method can be used [22]. Here every sensor monitor has one hop neighbors.

We can see that the physiological parameters are largely correlated in health monitoring. So in this framework the proposed approach can increase the overall accuracy of the anomaly detection system. Here the local as well as temporal dependencies exploited over the parameters. It helps to find the different between faulty measurement and temporal dependencies. A purpose of the system is to give surety over reliable operations of sensors and also made a perfect result over medical diagnosis. In every case, there find a correlation between time and space for sensor measurements. Here errors are not correlated with other subjects.

In this paper, a simple, adaptable and intelligent approach is proposed for false alarm detection in a wireless body sensor network. The proposed approach is based on MD and Dynamic Threshold Calculation (DTC). And the desired objective of our approach is to reduce false alarm which occurs for the 
faulty measurement as well as promoting the credibility and the appropriateness of the monitoring system.

\section{BACKGROUND}

In our framework we used both of MD and the Dynamic Threshold Calculation (DTC). Here in this section we try to review both of these concepts shortly.

\subsection{Mahalanobis Distance}

MD is used both over measured and received multivariate instance for detecting anomalous. To exploit promiscuous monitoring a single parameter by placing redundant sensors is impractical for medical applications. Healthy and unhealthy electronic products can be classified by MD [20].

Mahalanobis Distance[40] or MD is a very common method which is used mostly for the outlier detection over multivariate data.

In the mathematical glance, if we proposed a multivariate data $\mathrm{X}$;

Where, $\mathrm{X}=\left(A_{1}, A_{2}, A_{p}\right)$.

Here,

$$
A_{k}=\left(X_{1 k}, X_{2 k}, \ldots \ldots, X_{n k}\right)
$$

is a set of $n$ observations of the $k$ th attribute, and Xi represent an instance vector $X_{i}=\left(x_{i 1}, x_{i 2}, \ldots, x_{i p}\right)$ [28].

$$
\begin{gathered}
A_{1} \ldots A_{p} \\
X=\begin{array}{c}
X_{1} \\
\vdots \\
X_{n}
\end{array}\left(\begin{array}{ccc}
x_{11} & \cdots & x_{1 p} \\
\vdots & \ddots & \vdots \\
x_{n 1} & \cdots & x_{n p}
\end{array}\right)
\end{gathered}
$$

The distance between attributes can be measured by the MD. Here the correlation between them is taken into the account.

$$
\sqrt{\left(X_{i}-\mu\right)^{T} \sum^{-1}\left(X_{i}-\mu\right)} ; \text { where }, \mu=(\mu 1, \mu 2, \ldots, \mu p)^{T}
$$

It is the mean vector $(1 \times p)$ and is the covariance matrix $(p \times p)$ of these $\mathrm{p}$ attributes, calculated as:

$$
\begin{gathered}
\mu_{k}=\frac{1}{n} \sum_{i=1}^{n} x_{i k} \\
\sum_{i f}=\frac{1}{n-1} \sum_{k-1}^{u}\left(x_{k i}-\mu_{i}\right)\left(x_{k j}-\mu_{j}\right)
\end{gathered}
$$

If the value of MDi is being large, that means there is a large detachment between attributes.

\subsection{Dynamic Threshold Calculation}

Another approach is used over WSNs called Dynamic Threshold Calculation (DTC)[38]. Here in physiological parameters, the prognostic can start for the future next value by using the past value. The calculation of error can be possible here with the difference the desire sensed value and the predicted value. Here dynamic threshold based error computation is used in order to detect anomalies over medical healthcare on body sensor.
Here the prognostic can start for the future next value by using the past value, this happened to the physiological parameters. As soon as the storage node or a sink node can able to receive the desired sensor data, the prediction can be possible. We know, in this system the storage nodes [9] are a special kind of nodes where includes higher storage as well as processing capability. In this case, the cost of storage and processes are not able to affect the whole network due to energy expenditure. Eventually the actual sensor data are received by the storage node and also these data are being compared with the predicted value. The calculation of error can be possible here with the difference the desire sensed value and the predicted value. With the calculation, we get that the total error in the particular parameter is less than its threshold value, then the actual values will be updated with the predicted values. To this point, the system will be prepared for the next prediction. For every parameter, it is possible to determine the threshold value under consideration with its statistical analysis based on the subjects past or historical data. On the other hand, for a particular physiological parameter if the error is greater than the threshold value, then that parameter will be correlated with other physiological parameters. In this process an algorithm is used which we described in the next section.

We can see that most of the medical conditions have a number of parameters such as BP, Pulse, HR, Respiration, Spo2 and so on. These all are varied with a correlated manner. So the determination of the parameters abnormal values can be justified here, which based on the other variant related physiological parameter. On other case, if it is not possible, then an anomalous identification will be granted for the collected parameter as well as any kind of alarm can be declared here as a false alarm.

\section{SOLUTION OR PROPOSED APPROACH}

A general medical deployment scenario is considered here, where $\mathrm{N}(N \leq p)$ wireless nodes $(S 1, \ldots, S N)$ with restricted resources are placed on the patients body (as shown in Fig. 1). These sensors 29] are used to collect vital signs and transmit the collected data at regular time interval to asink device. Faulty measurements must be detected and isolated in order to reduce false alarms and prevent fault diagnosis.

Sensor data are sent to the server through the wireless sensor

$$
\text { Client Server }
$$

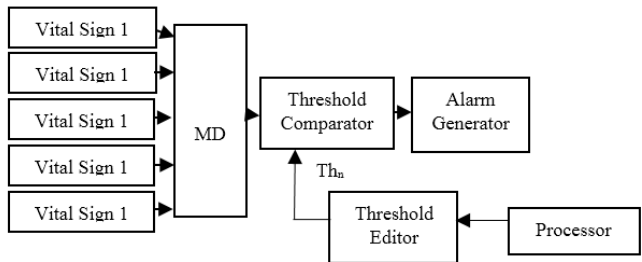

Fig 2. Wireless Body Sensor Network

network for processing. Five different sensor data are used for the processing. They are Blood Pressure, Heart Rate, Pulse, Respiration Rate, Oxygenation Rate for the simulation.

Weve considered a threshold value for the correlation measurement with the input attribute from the sensor nodes. The threshold value is calculated by the following equation: 


$$
T h=\left\{\begin{array}{l}
\text { Th, if AlarmisTY } \\
\text { Th+k, if AlarmisTN } \\
\text { Th, if AlarmisFY } \\
\text { Th }-k, \text { if AlarmisFN }
\end{array}\right.
$$

Where, $\mathrm{K}=\frac{|T h-F P|}{\mid \text { Min-Max }}$

Where, $\mathrm{Th}=$ Threshold Value, Min = Recent Minimum Peak Value, $\mathrm{Max}=$ Recent Maximum Peak Value. $\mathrm{FP}=$ The peak value where the alarm result is not true means, the alarm needed to trigger but it was not triggered or vice-versa. TY stands for the alarm which is triggered when its necessary. TN means the alarm is triggered when it is not necessary. FY means the alarm is not triggered and its true. FN mens the alarm is not triggered when it is necessary.

The methodology for the proposed Dynamic Threshold Calculator [17] flowchart is shown in Fig 3. When the new instance

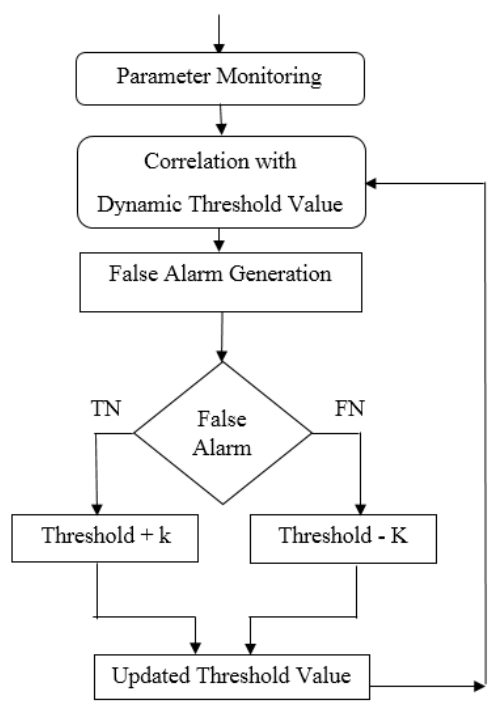

Fig 3. Flow Chart of Dynamic Threshold Calculation

is arriving, MD is calculated between the sliding window and the training data and the current attribute values. The dynamic threshold calculation, then take place for measuring the threshold values. Then the correlation started between the input attributes of the sensor and the threshold value. When the input attributes are greater than the threshold value then the alarm is generated. After the generation of the alarm it is processed for whether it is a false positive or a false negative alarm. This situation is handled by an algorithm of dynamic threshold calculation which is shown in the Fig 3.

\section{EXPERIMENTAL APPROACH}

Real medical data in sensor nodes are used to build a Wireless Body Sensor Network to implement the calculation. In
PhysioNet database (MIMIC Database) [3] real clinical health data can be found. In total seven attributes are used. Those are blood pressure (BPmean) mean values, systolic blood pressure, diastolic blood pressure, HR, pulse, respiration rate (RESP), and oxygenation ratio (SpO2). But the focus was mainly in five attributes $(\mathrm{p}=5)$. And those attributes are $\mathrm{SpO} 2$, BPmean, pulse, HR, and RESP. As far as our knowledge those datasets were faultless.

In Fig 4 to 8 the variations of BPmean, HR, pulse, RESP, and

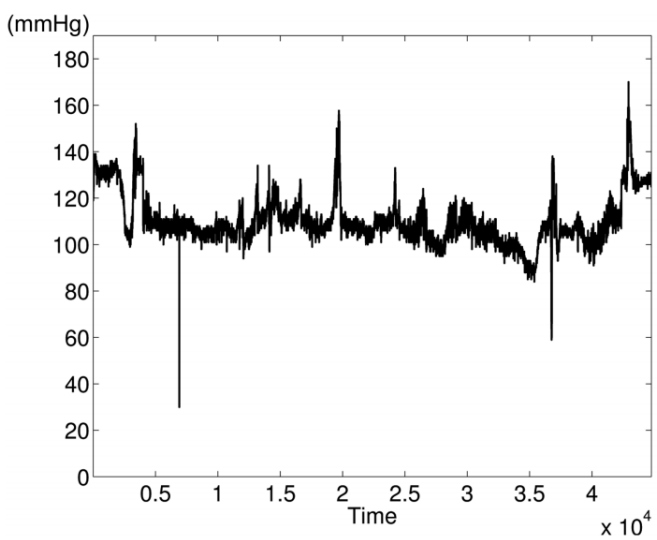

Fig 4. Blood Pressure

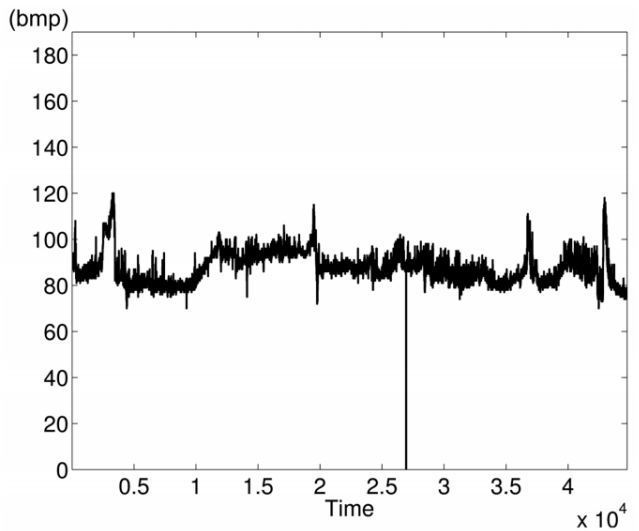

Fig 5. Heart Rate

$\mathrm{SpO} 2$ are presented in respectively. BP is measured with normal values $(\in[90140]$ and its unit is in millimeters of mercury $(\mathrm{mmHg})$. Beats per minute $(\mathrm{bpm})$ unit is used for HR and pulse presentation with the age of [60-100] normal healthy persons. The $\mathrm{SpO} 2$ is measured in percentage and the Respiration per minute (rpm) is used for RESP of oxygen in the blood with respect to normal values $\in[95 \%-100 \%]$.

As all the people are not same, so their physiological parameters will be different and they depend on sex, age, weight, activity, etc parameters. We used static interval for anomaly detection, which heavily depends on many additional dynamic parameters such as environmental, ages, activities: rest, moving, awake, sleep, etc. These parameters are difficult to set in a dynamic way.

In fig. 5, we can observe some change of BP falling to 30 and $55 \mathrm{bpm}$. Furthermore, in Figs. 4 and 5 some HR and pulse value 


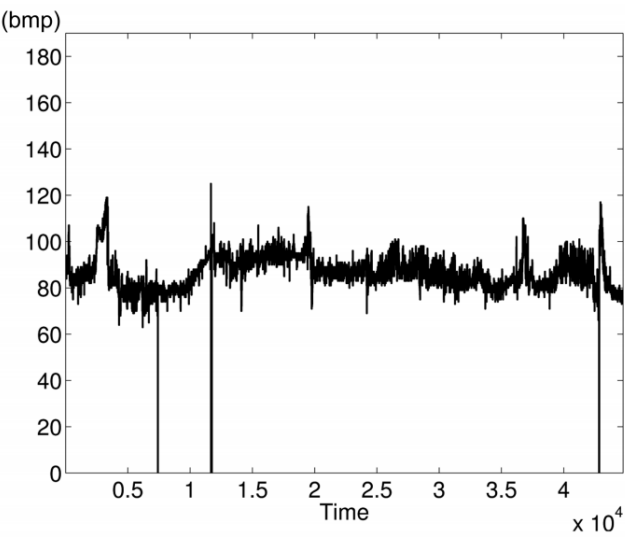

Fig 6. Pulse

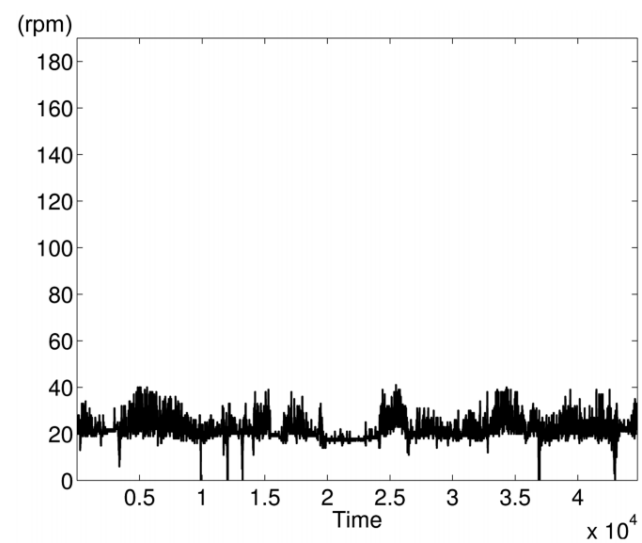

Fig 7. Respiration Rate

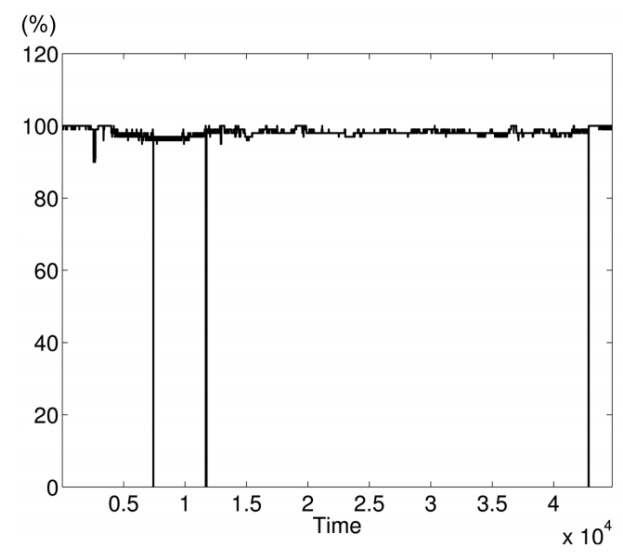

Fig 8. Oxygenation Rate

decreases zero in different time intervals. We can also observe some abnormal variation in RESP and the $\mathrm{SpO} 2$ in Fig 7 and 8. In Fig 9 we show a variation curve to prove the correlation between monitor attributes. In this figure it can be seen that at the same time instant clinical emergency induces some change in many parameters. Among monitored attributes, there is no spatial correlation of faulty measurements, where one of the

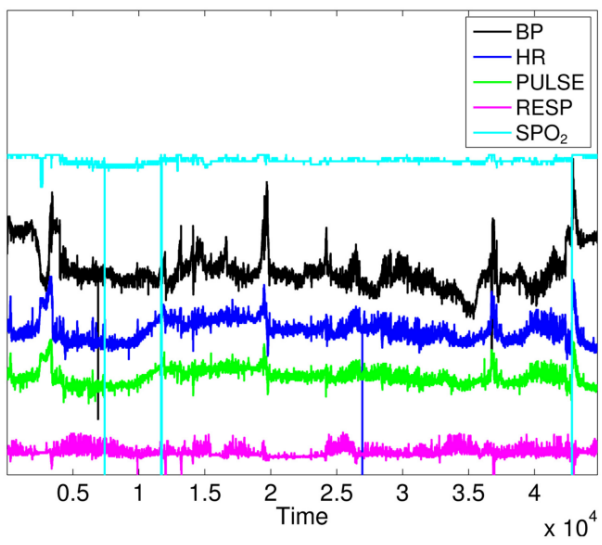

Fig 9. All Parameter

attributes independently change from others. For clearing the shape of their variations some variation curves are shifted in fig 8 . There are four changes those can be seen clearly in the figure, where some attributes value increases and some decreases.

After getting the physiological attributes of the sensor node via

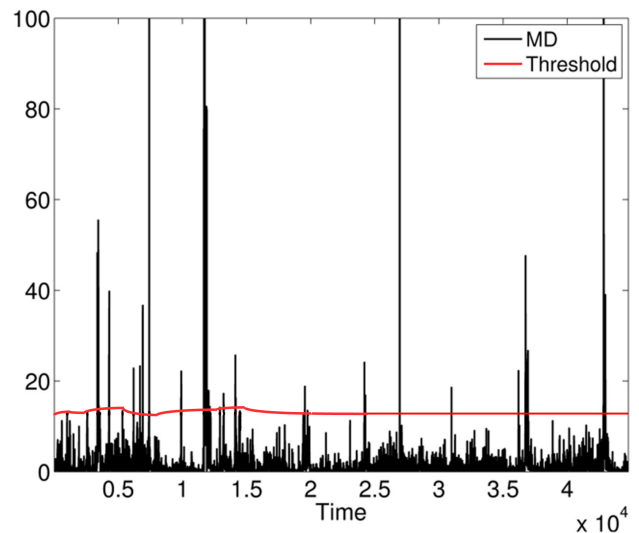

Fig 10. squared Mahalanobis distance (MD) and threshold

the wireless sensor network it is necessary to calculate the MD over them. Then the dynamic threshold calculation algorithm took place and measure an idle threshold for the attributes. Those tasks are presented in Fig 10.

\section{SIMULATION}

This paper uses MATLAB and Simulink with Truetime toolbox[11] for simulating the process. We use Zigbee[8] protocol for simulating Wireless Sensor Network (WSN).

\section{SIMULATION TOOLS}

\subsection{Node/sensor Data}

The heading of subsections should be in Times New Roman 12-point bold with only the initial letters capitalized. (Note: For subsections and subsubsections, a word like the or a is not capitalized unless it is the first word of the header.) 


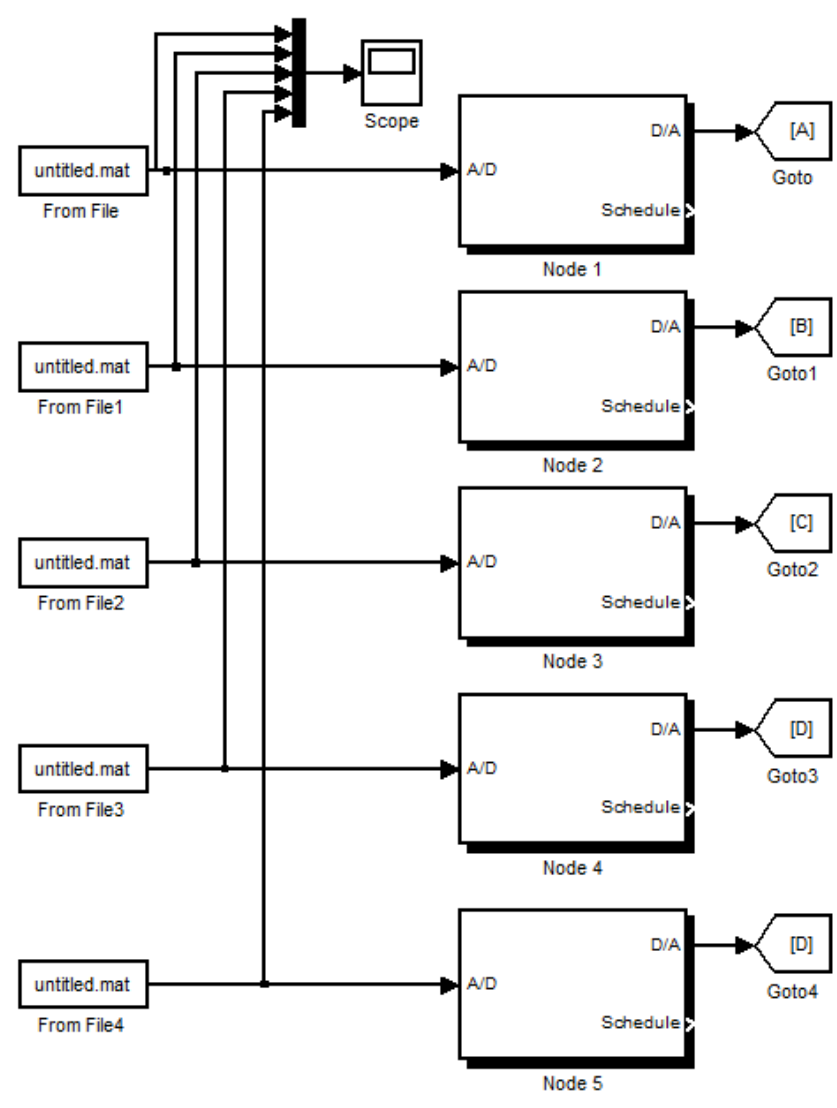

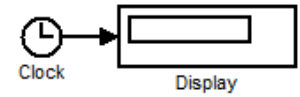

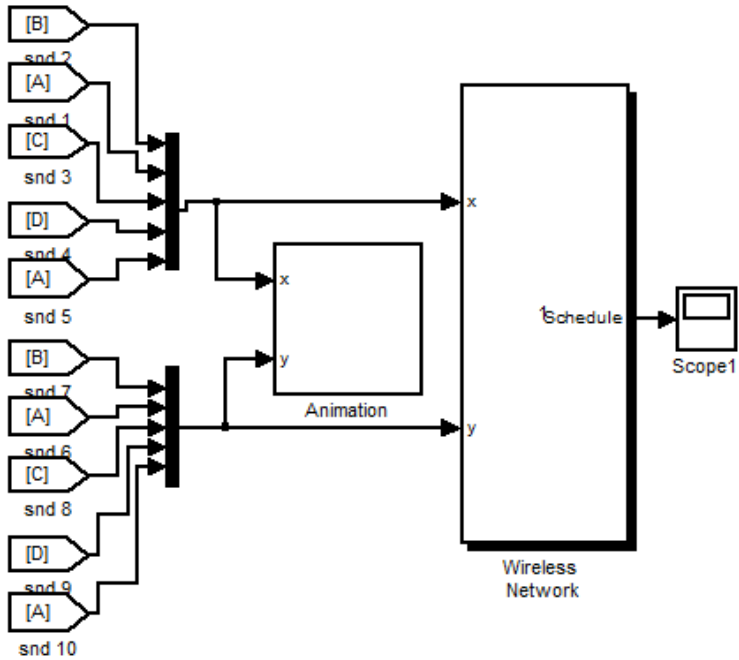

Fig 11. Simulation

7.1.1 PhysioBank. PhysioBank is a kind of multi-parameter database that provides biomedical research related digital database. We used the biomedical sensor database from this database website. PhysioBank database includes header file (.hea), signal files (.dat) and annotation files (.atr or .al). As this database use UNIX development environment, we cant use the data directly. For using this database we have to convert the files using WFDB toolbox.

7.1.2 WFDB Toolbox. For reading and analyzing PhysioBank data, we used this toolbox. It has WFDB library, WFDB applications and WAVE for reading and writing signal files.

\subsection{Matlab/Simulink}

MATLAB is a high-level programming language and interactive environment that is used for algorithm development, data visualization [30], data analysis and numerical calculation. In this paper, we used MATLAB Simulink software with Truetime toolbox for simulating Wireless Body Sensor Network[7].

\subsection{Truetime Toolbox}

Truetime [2] is a freeware MATLAB library for simulating networked and embedded real time control system.

\section{RESULT}

In this section, the performance analysis results of the proposed approach for anomaly detection in medical WSN are presented. Afterward, some analysis are conducted to study the impact of the decision threshold on true positive and false alarm ratio.

To evaluate the performance of the proposed approach, some abnormal values were injected at different time instants in the different attributes. Then the receiver operating characteristic (ROC)[42] curve is used to analyze the impact of detection threshold (h) on the detection accuracy and the false alarm ratio. The ROC curve is used for accurate analysis when varying the value of the decision threshold. In general, a good detection algorithm must achieve a high detection ratio with the lowest false alarm rate. 


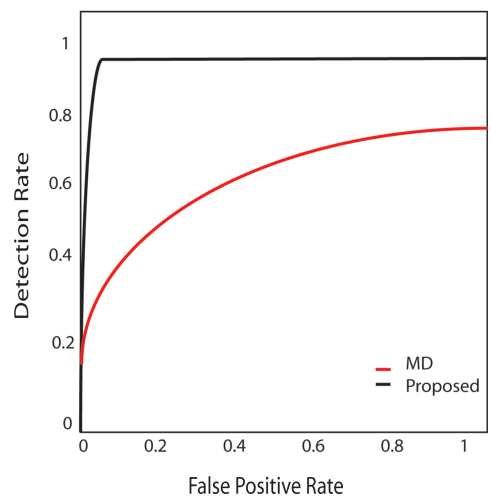

Fig 12. The receiver operating characteristics

The ROC curve presented in Fig 12 shows the relationship between the detection rate and the false alarm rate.

$$
\text { Detectionrate }=\frac{T P}{T P+F N}
$$

Where TP is the number of true positives and FP the number of false positives. The false positive rate is defined as:

$$
\text { Falsepositiverate }=\frac{F P}{F P+T N}
$$

As existing anomalies are not enough to realize this analysis, we synthetically injected 100 anomalies at known time instants in the used dataset. A good detection mechanism should achieve a high detection ratio with the lowest false alarm rate. Fig. 11 shows that our proposed framework can achieve a DR of $98 \%$ with an FPR of $2.3 \%$

\section{FUTURE WORK}

In our framework, as all of the processes over data are working through the server site. As we know that there are required a high bandwidth for the transfer of data from sensor to server site at every process. In future we hope to update our approach at the transfer process of data, where all the activities have taken place over the node as well as a sensor, there will be no need to go through the server. As a result, it can require a very low bandwidth.

In most anomaly detection processes, all kinds of actual sensor data as well as prediction data have to go through from node or sensor to the server site. This is obviously a time consuming process. We are trying to include a microcontroller with a very little processing power in our system. And adjust this microcontroller with the node or sensor for every data transmission process. Then this overall process can be able to decrease the complexity of time swallowing.

The path of data transference from the sensor to the server is very much critical. It has a possibility of missing any valuable data between the connection of the server and sensor. Just any single corrupted or missing data can able to make a revolutionary changed in the overall result, which is one kind of impendance not only for the medial domain but also for a patient. In future,our aim is to propose an update framework over a structural transference system where, data accuracy, efficiency as well as a smart alarm system must be required.

\section{REFERENCES}

[1] Crossbow Technology, Inc. MICAz ZigBee Series (MPR2400)., 2011(accessed 13-Nov- 2014). http://www.oalib.com/references/9250153

[2] Automatic Control - TrueTime., (accessed 13-Nov- 2014). http://www. control.lth.se/truetime

[3] PhysioBank ATM., (accessed 13-Nov- 2014). https:// physionet.org/cgi-bin/atm/ATM

[4] CodeBlue: Wireless Sensors for Medical Care - Harvard Sensor Networks Lab (fiji.eecs.harvard.edu)., (Accessed: 13-Nov-2015 ). http://www.webinfobits.com/page/ c47aea7841ef.

[5] The Sensor Network Museum: Projects - Tmote Sky browse., (Accessed: 13-Nov-2015). http://www.snm. ethz.ch/Projects/TmoteSky

[6] Hande Alemdar and Cem Ersoy. Wireless sensor networks for healthcare: A survey. Computer Networks, 54(15):2688-2710, 2010.

[7] Djamel Benferhat, Frédéric Guidec, and Patrice Quinton. Cardiac monitoring of marathon runners using disruptiontolerant wireless sensors. In Ubiquitous Computing and Ambient Intelligence, pages 395-402. Springer, 2012.

[8] Christopher M Bishop. Pattern recognition and machine learning. springer, 2006.

[9] T Ryan Burchfield and Subbarayan Venkatesan. Accelerometer-based human abnormal movement detection in wireless sensor networks. In Proceedings of the 1st ACM SIGMOBILE international workshop on Systems and networking support for healthcare and assisted living environments, pages 67-69. ACM, 2007.

[10] Adrian Burns, Barry R Greene, Michael J McGrath, Terrance J O'Shea, Benjamin Kuris, Steven M Ayer, Florin Stroiescu, and Victor Cionca. Shimmer-a wireless sensor platform for noninvasive biomedical research. Sensors Journal, IEEE, 10(9):1527-1534, 2010.

[11] Alberto Cardoso, Sérgio Santos, Amâncio Santos, and Paulo Gil. Simulation platform for wireless sensor networks based on the truetime toolbox. In Industrial Electronics, 2009. IECON'09. 35th Annual Conference of IEEE, pages 2115-2120. IEEE, 2009.

[12] Xu Cheng, Ji Xu, Jian Pei, and Jiangchuan Liu. Hierarchical distributed data classification in wireless sensor networks. Computer Communications, 33(12):1404-1413, 2010.

[13] Jongyoon Choi, Beena Ahmed, and Ricardo GutierrezOsuna. Development and evaluation of an ambulatory stress monitor based on wearable sensors. Information Technology in Biomedicine, IEEE Transactions on, 16(2):279-286, 2012.

[14] Arnoldo Díaz-Ramírez, Francisco A Bonino, and Pedro Mejía-Alvarez. Human detection and tracking in healthcare applications through the use of a network of sensors. In $\mathrm{Hu}$ man Behavior Understanding in Networked Sensing, pages 171-190. Springer, 2014.

[15] Pedro Forero, Alfonso Cano, Georgios B Giannakis, et al. Distributed clustering using wireless sensor networks. Selected Topics in Signal Processing, IEEE Journal of, 5(4):707-724, 2011. 
[16] Krešimir Grgić, Drago Žagar, and Višnja Križanović. Medical applications of wireless sensor networks-current sta-tus and future directions. Official Publication of the Medical Association of Zenica-Doboj Canton Bosnia and Herzegovina, page 23, 2011.

[17] Shah Ahsanul Haque, Mustafizur Rahman, and Syed Mahfuzul Aziz. Sensor anomaly detection in wireless sensor networks for healthcare. Sensors, 15(4):8764-8786, 2015.

[18] Fei Huang, Zhipeng Jiang, Sanguo Zhang, and Suixiang Gao. Reliability evaluation of wireless sensor networks using logistic regression. In Communications and Mobile Computing (CMC), 2010 International Conference on, volume 3, pages 334-338. IEEE, 2010.

[19] JeongGil Ko, Jong Hyun Lim, Yin Chen, Rvăzvan Musvaloiu-E, Andreas Terzis, Gerald M Masson, Tia Gao, Walt Destler, Leo Selavo, and Richard P Dutton. Medisn: Medical emergency detection in sensor networks. ACM Transactions on Embedded Computing Systems (TECS), 10(1):11, 2010.

[20] Sachin Kumar, Tommy WS Chow, and Michael Pecht. Approach to fault identification for electronic products using mahalanobis distance. Instrumentation and Measurement, IEEE Transactions on, 59(8):2055-2064, 2010.

[21] Ye Li, Yongli Wang, and Guoping He. Clustering-based distributed support vector machine in wireless sensor networks. Journal of Information \& Computational Science, 9(4):1083-1096, 2012.

[22] Fang Liu, Xiuzhen Cheng, and Dechang Chen. Insider attacker detection in wireless sensor networks. In INFOCOM 2007. 26th IEEE International Conference on Computer Communications. IEEE, pages 1937-1945. IEEE, 2007.

[23] David Malan, Thaddeus Fulford-Jones, Matt Welsh, and Steve Moulton. Codeblue: An ad hoc sensor network infrastructure for emergency medical care. In International workshop on wearable and implantable body sensor networks, volume 5, 2004.

[24] Aleksandar Milenković, Chris Otto, and Emil Jovanov. Wireless sensor networks for personal health monitoring: Issues and an implementation. Computer communications, 29(13):2521-2533, 2006

[25] K Montgomery, C Mundt, G Thonier, A Tellier, U Udoh, V Barker, R Ricks, L Giovangrandi, P Davies, Y Cagle, et al. Lifeguard-a personal physiological monitor for extreme environments. In Engineering in Medicine and Biology Society, 2004. IEMBS'04. 26th Annual International Conference of the IEEE, volume 1, pages 21922195. IEEE, 2004.

[26] Karla Felix Navarro, Elaine Lawrence, and Brian Lim. Medical motecare: A distributed personal healthcare monitoring system. In eHealth, Telemedicine, and Social Medicine, 2009. eTELEMED'09. International Conference on, pages 25-30. IEEE, 2009.

[27] Sutharshan Rajasegarar, Christopher Leckie, James C Bezdek, and Marimuthu Palaniswami. Centered hyperspherical and hyperellipsoidal one-class support vector machines for anomaly detection in sensor networks. Information Forensics and Security, IEEE Transactions on, 5(3):518-533, 2010

[28] Osman Salem, Alexey Guerassimov, Ahmed Mehaoua, Andrian Marcus, and Borko Furht. Sensor fault and patient anomaly detection and classification in medical wireless sensor networks. In Communications (ICC), 2013 IEEE International Conference on, pages 4373-4378. IEEE, 2013.

[29] Osman Salem, Yaning Liu, Ahmed Mehaoua, and Raouf Boutaba. Online anomaly detection in wireless body area networks for reliable healthcare monitoring. Biomedical and Health Informatics, IEEE Journal of, 18(5):15411551,2014

[30] Gursel Serpen, Jiakai Li, and Linqian Liu. Ai-wsn: Adaptive and intelligent wireless sensor network. Procedia Computer Science, 20:406-413, 2013.

[31] Faizah Shaari, Azuraliza Abu Bakar, and Abdul Razak Hamdan. A predictive analysis on medical data based on outlier detection method using non-reduct computation. In ADMA, pages 603-610. Springer, 2009.

[32] Nauman Shahid, Ijaz Haider Naqvi, and Saad Bin Qaisar. Quarter-sphere svm: attribute and spatio-temporal correlations based outlier \& event detection in wireless sensor networks. In Wireless Communications and Networking Conference (WCNC), 2012 IEEE, pages 2048-2053. IEEE, 2012.

[33] Abhishek B Sharma, Leana Golubchik, and Ramesh Govindan. Sensor faults: Detection methods and prevalence in real-world datasets. ACM Transactions on Sensor Networks (TOSN), 6(3):23, 2010.

[34] Bo Sheng, Qun Li, Weizhen Mao, and Wen Jin. Outlier detection in sensor networks. In Proceedings of the 8th ACM international symposium on Mobile ad hoc networking and computing, pages 219-228. ACM, 2007.

[35] Supakit Siripanadorn, Wipawee Hattagam, and Neung Teaumroong. Anomaly detection in wireless sensor networks using self-organizing map and wavelets. International Journal of Communications, 4(3):74-83, 2010.

[36] Qingquan Sun, Fei Hu, and Qi Hao. Mobile target scenario recognition via low-cost pyroelectric sensing system: Toward a context-enhanced accurate identification. Systems, Man, and Cybernetics: Systems, IEEE Transactions on, 44(3):375-384, 2014.

[37] Sergios Theodoridis, Aggelos Pikrakis, Konstantinos Koutroumbas, and Dionisis Cavouras. Introduction to Pattern Recognition: A Matlab Approach: A Matlab Approach. Academic Press, 2010.

[38] Anthony Wood, Gilles Virone, Thao Doan, Quihua Cao, Leo Selavo, Yafeng Wu, L Fang, Zhimin He, Shan Lin, and Jack Stankovic. Alarm-net: Wireless sensor networks for assisted-living and residential monitoring. University of Virginia Computer Science Department Technical Report, 2, 2006.

[39] Yan Xiaozhen, Xie Hong, and Wang Tong. A multiple linear regression data predicting method using correlation analysis for wireless sensor networks. In Cross Strait Quad-Regional Radio Science and Wireless Technology Conference (CSQRWC), 2011, volume 2, pages 960-963. IEEE, 2011.

[40] Miao Xie, Song Han, Biming Tian, and Sazia Parvin. Anomaly detection in wireless sensor networks: A survey. Journal of Network and Computer Applications, 34(4):1302-1325, 2011.

[41] Suya Xu, Caiping Hu, Lisong Wang, and Guobin Zhang. Support vector machines based on k nearest neighbor algorithm for outlier detection in wsns. In Wireless Coтmunications, Networking and Mobile Computing (WiCOM), 2012 8th International Conference on, pages 1-4. IEEE, 2012.

[42] Xiuxin Yang, Anh Dinh, and Li Chen. Implementation of a wearerable real-time system for physical activity recognition based on naive bayes classifier. In Bioinformatics and Biomedical Technology (ICBBT), 2010 International Conference on, pages 101-105. IEEE, 2010.

[43] Yang Zhang, Nirvana Meratnia, and Paul Havinga. Adaptive and online one-class support vector machine-based outlier detection techniques for wireless sensor networks. 
Communications on Applied Electronics (CAE) - ISSN : 2394 - 4714

Foundation of Computer Science FCS, New York, USA

Volume 3 - No. 6, December 2015 - www.caeaccess.org

In Advanced Information Networking and Applications Workshops, 2009. WAINA'09. International Conference on, pages 990-995. IEEE, 2009.

[44] Yang Zhang, Nirvana Meratnia, and Paul Havinga. Outlier detection techniques for wireless sensor networks: A survey. Communications Surveys \& Tutorials, IEEE, 12(2):159-170, 2010. 